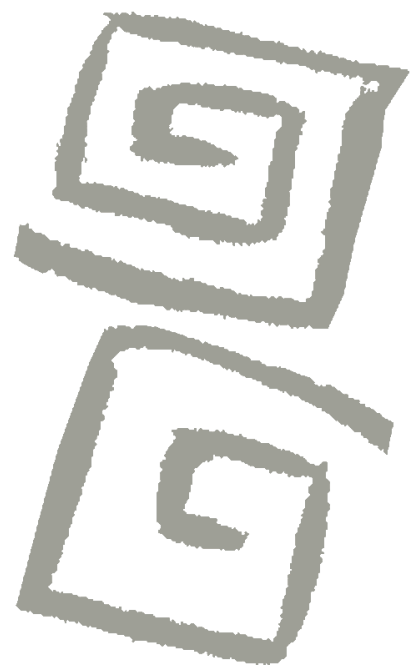

\title{
El saber ver y el saber hacer en el hospital-asilo de Oliva (1914-1950): el caso Adela
}

\author{
Know-what and know-how in the Hospital-Asilo of \\ Oliva (1914-1950): the Adela case
}

Laura Natalia Vanadia', María Laura Rodríguez ${ }^{2}$

'Licenciada en Psicología. Doctoranda, Doctorado en Ciencias de la Salud Facultad de Ciencias Médicas, Universidad Nacional de Córdoba. Becaria doctoral, Consejo Nacional de Investigaciones Científicas y Técnicas, Córdoba, Argentina. $₫$ (iD

2Licenciada en Historia. Doctora en Ciencias Políticas. Investigadora adjunta, Consejo Nacional de Investigaciones Científicas y Técnicas, Córdoba, Argentina. $₫$ (iD)
RESUMEN En este trabajo analizamos múltiples experiencias en el funcionamiento del primer open-door creado en la provincia de Córdoba, en 1914: el "Asilo Colonia Regional Mixto de Alienados Oliva". Abordamos un conjunto de historias clínicas y publicaciones especializadas del boletín institucional, profundizando en procesos teóricos y terapéuticos articulados durante la primera década de internación de una paciente que vivió en Oliva la mayor parte de su vida adulta. Este estudio de caso, cuestiona la tradicional relevancia otorgada a este manicomio como espacio de reclusión y control social. Mostramos que, en un contexto de constante crisis de los recursos institucionales y de trasformación de los modelos psiquiátricos, la configuración del "saber ver" y el "saber hacer" de estos alienistas no constituyeron una autoridad monolítica, dando cuenta de cómo los comportamientos de los pacientes y sus allegados, los valores y usos sociales definían tipos de ingresos, diagnósticos y posibilidades de tratamiento.

PALABRAS CLAVES Psiquiatría; Historia; Hospitales Psiquiátricos; Trastornos Mentales; Argentina.

ABSTRACT This paper analyze multiple experiences about the functioning of the first Open-Door in Argentina's inside, the "Asilo Colonia Regional Mixto de Alienados Oliva", created in the province of Cordoba in 1914. We approach a set of clinical histories and specialized publications of the institutional bulletin, deepening in theoretical and therapeutic processes articulated during the first decade of hospitalization of a patient who lived in Oliva most of her adult life. This study case questions the relevance given to this madhouse as a space of reclusion and social control. We show that, in a context of crisis about institutional resources and transformation into psychiatric models, the configuration of know-what and know-how of these alienists did not constitute a monolithic authority, accounting for how the behaviors of patients and their relatives, values and social uses defined types of income, diagnoses and treatment possibilities.

KEY WORDS Psychiatry; History; Hospitals, Psychiatric; Mental Disorders; Argentina. 


\section{INTRODUCCIÓN}

Como en otras partes de América Latina, en Argentina, la creación de asilos y hospitales regionales para indigentes fue una aspiración planteada desde fines del siglo XIX, cuando los médicos comenzaron a denunciar el estado de abandono que sufrían los pacientes, situación que, en nuestro país, se sumaba a los ribetes propios de la acelerada modernización socioeconómica y del crecimiento demográfico en marcha.

La incorporación del país al mercado internacional y su adaptación al modelo agroexportador, en el marco de la división internacional del trabajo, transformó no solo la organización productiva, sino también el orden social ${ }^{(1)}$. A pesar de que la expansión demográfica beneficiaría a la economía del país, rápidamente dejó traslucir importantes consecuencias indeseables, como el incremento de la pobreza urbana, el hacinamiento, la delincuencia y la propagación de enfermedades $^{(2,3)}$. Los ideales modernos de "orden" y "progreso", pilares en la formación del Estado, requerían de la erradicación de los males urbanos integrados por la marginalidad, la delincuencia y la locura.

Como sostiene Ferrari ${ }^{(4)}$, en este contexto, los médicos higienistas habilitaron espacios de intervención, convirtiéndose en administradores de asilos y hospicios, en los que de manera paulatina se desplazó la participación de otros actores, como las sociedades de beneficencia, en pos de un discurso científico positivista que se consolidaba a través de la creación de cátedras y el acceso de la elite médica a espacios universitarios y hospitalarios. Las modalidades de intervenir la locura no perderían de vista el ideal de hombre moral, la defensa de la raza nacional y la construcción del buen ciudadano ${ }^{(4,5,6)}$. El proyecto presentado al senado en 1906 y aprobado en la presidencia de Figueroa Alcorta -Ley $4953^{(5)}$ - fue el impulso legal para un modelo hospitalario añorado por muchos. Basado en los principios del no restraint, tratamientos en espacios rurales y "...procedimientos más liberales y humanitarios de asistencia", estaba destinado a la atención de procesos agudos y a dar albergue a la mayor cantidad de enfermos crónicos e incurables ${ }^{(5)}$. En ese contexto, las gestiones del doctor Domingo Cabred fueron decisivas para la creación del "Asilo Colonia Regional Mixto de Alienados Oliva", inaugurado en la provincia de Córdoba en $1914^{(7)}$, bajo la modalidad de institución open door.

De acuerdo a la importancia estratégica que se le reconoció al Asilo Colonia de Oliva, el más grande del país y el primero de la provincia de Córdoba, dicha institución se convirtió tempranamente en objeto de la historiografía de la psiquiatría argentina. Una serie de investigaciones producidas entre fines de la década de 1990 y comienzos de la década siguiente plantearon que la configuración institucional de Oliva daba cuenta de un lugar caracterizado por la segregación y el disciplinamiento, donde el enfermo mental crónico era conceptuado más bien como un medio de sostenimiento económico institucional, separado de la intervención disciplinar estrictamente psiquiátrica ${ }^{(7,8,9)}$.

Estas aproximaciones, vinculadas a la renovación de las perspectivas historiográficas clásicas, generada a partir de los aportes de Vezzetti(6), enfatizaron cuestiones relativas al rol de las instituciones psiquiátricas como dispositivos de poder articulados al servicio del Estado. Sin embargo, lecturas más recientes, como las de Ablard $^{(10)}$, pusieron en tensión aquellas aseveraciones sobre la existencia de un todopoderoso Estado nacional, con capacidad de intervención y regulación absoluta sobre los cuerpos y voluntades de los pacientes ${ }^{(11)}$.

La historiografía latinoamericana viene insistiendo en que el estudio más detallado de las diversas fuentes relativas a la admisión y tratamiento de alienados, rescata nuevas evidencias sobre la existencia de una preocupación y de una práctica médica dirigida a los alienados, en contraposición con la visión que lo consideraba como mero lugar de exclusión social| ${ }^{(8,12)}$. Si bien en el contexto de América Latina, y de Argentina en particular, el proceso de atención en salud mental se vio obstaculizado por el débil apoyo estatal 
y la falta de recursos para hacer frente a la creciente demanda de atención, el manicomio fue un espacio de múltiples experien$\operatorname{cias}^{(12,13,14,15)}$. Desde esta perspectiva, cada vez son más frecuentes los trabajos que destacan el carácter social híbrido y polifacético del manicomio, a partir de analizar su vida cotidiana, proponiendo un diálogo entre las distintas voces y sujetos que lo habitaron ${ }^{(16)}$.

A partir de este enfoque vinculado a una historia social de la psiquiatría, abordamos un conjunto de historias clínicas y publicaciones especializadas aparecidas en el Boletín del Asilo de Alienados de Oliva, centrándonos en la experiencia que atravesó Adela, paciente que vivió y murió en la institución luego de pasar 38 años de internación. En este estudio de caso, ponemos en juego tres puntos de vista. En el primer apartado, recuperamos algunos renglones ligados a las circunstancias de la llegada de Adela a Oliva, a través de interrogar algunos datos e indicios sobre su perfil social y familiar, así como en cuanto a lógicas heterogéneas de los ingresos y procesos de atención. En el segundo y tercer apartado, procuramos tensionar las relaciones entre saber/poder, problematizando la práctica especializada alrededor de lo que hemos denominado "el saber ver" y "el saber hacer" de los alienistas, para lo cual identificamos y ubicamos en contexto científico las dinámicas involucradas en la construcción del diagnóstico, atendiendo tanto a las elaboraciones teóricas, aportes y trasformaciones, como a la porosidad de ese saber. Finalmente, analizamos la terapia del trabajo o laborterapia como eje del funcionamiento institucional y de la praxis clínica en Oliva y exponemos cómo las ineficacias terapéuticas ya reconocidas, coexistieron con otras complejidades, tanto las propias del caso de Adela, como las vinculadas a tramas científicas e institucionales clave para examinar el funcionamiento institucional.

Las posibilidades y obstáculos ligados al uso de las historias clínicas como fuente merecen un apartado particular. Sin duda, su valor heurístico es sumamente auspicioso, no solo habilita un acceso directo a la práctica médica, sino además ofrece información para emprender otros tipos de análisis, entrando de lleno en el campo de la historia social $^{(17)}$. No obstante, esta misma amplitud de horizontes nos lleva a reconocer como límites al estado de dispersión y descuido en que hallamos los archivos específicos. Desde otra órbita de asuntos, a menudo nuestras fuentes clínicas abruman en silencios y vacíos, los que muchas veces fueron incorporados al análisis como inferencias indiciales. Ello también nos ha impelido a concentrar el análisis en la primera década de internación de Adela, aunque su permanencia en Oliva se extendió hasta 1969, año de su muerte, cuestiones que repasamos convenientemente.

Asimismo, aunque nuestra investigación, por su carácter histórico, trabaja con material de personas fallecidas en el siglo pasado, resulta necesario consignar que hemos manejado datos obtenidos de documentos personales. En consecuencia, en conformidad a la Ley 25326 sobre Protección de datos personales, se accedió al material asumiendo la responsabilidad de respetar la confidencialidad referida a la identidad de las personas mencionadas en las historias clínicas. Los nombres y todo dato filiatorio que podría llevar a su identificación son ficticios, por lo que, al citar expresiones textuales de las historias clínicas, solo se hará mención a las siglas de historia clínica (HC) y del Archivo del Hospital Emilio Vidal Abal (AHEVA) donde se encuentran alojadas las fuentes documentales, acompañadas del año.

\section{CUESTIONES DE FAMILIA}

El 15 de febrero de 1930, Adela reingresó a Oliva, comenzando una experiencia de vida que a la postre se puede analizar como definitiva, ya que concluyó con su muerte en la institución, en septiembre de 1969, 38 años después. Según los datos de su historia clínica, su primer internamiento, a cargo del Dr. Fazio, habría sido apenas unos meses antes, en 1929, con un carácter breve, ya que recibió su alta a los 20 días de su ingreso. Tanto el Dr. Fazio como el Dr. Barrancos -alienista 
que realizó su internación en 1930-, coincidieron en diagnosticarla con un cuadro de depresión melancólica simple. Cabe destacar que, además de estos dos ingresos, ella ya había pasado anteriormente 15 días en una institución administrada por las damas de la Sociedad de Beneficencia de Córdoba, conocida como "Asilo de Alienadas" de la ciudad de Córdoba (HC-AHEVA, 1929).

En términos generales, la historia clínica que se abrió en 1930 no abundó en datos personales no patológicos; no obstante, algunos de ellos son por demás significativos para trazar una suerte de perfil sociodemográfico de la paciente. Sabemos que su ingreso definitivo a la institución sucedió cuando ella tenía 20 años, siendo soltera, de profesión sirvienta, católica, sabía leer y escribir y, vivía con su padre, un italiano inmigrante, junto al que residía en la pequeña localidad rural de Arroyo Cabral, ubicada a 160 km de la ciudad de Córdoba.

Los alienistas que trabajaron con Adela sostuvieron silencios, que cobran un sentido clave para comprender la trayectoria que acabó en las internaciones de la paciente en Oliva. Algunos indicios sugieren que el contexto en que se produjo su internación, especialmente en lo que respecta a su entorno familiar y social, constituyeron piezas nodales para descubrir tramas sustanciales que habrían definido los procesos de ingreso y atención en las instituciones mentales públicas de estos años.

No era excepcional que quien solicitó la internación de la paciente fuera su propio padre, Antonio. Según marcó Sacristán ${ }^{(16)}$ era usual que las internaciones fueran solicitadas por la familia de los pacientes, por razones de preservación de la salud y vida, pero también por causas extra médicas. Resulta llamativo que, si bien su padre fue el interesado en que su hija fuera atendida en Oliva, Adela fue llevada a la institución por un amigo personal de este, Roque, quien para ello llevaba consigo una suerte de autorización ad hoc, dirigida al director del asilo, la que no era otra cosa que una carta manuscrita sin rubrica de ninguna autoridad, como puede verse en la Figura 1.

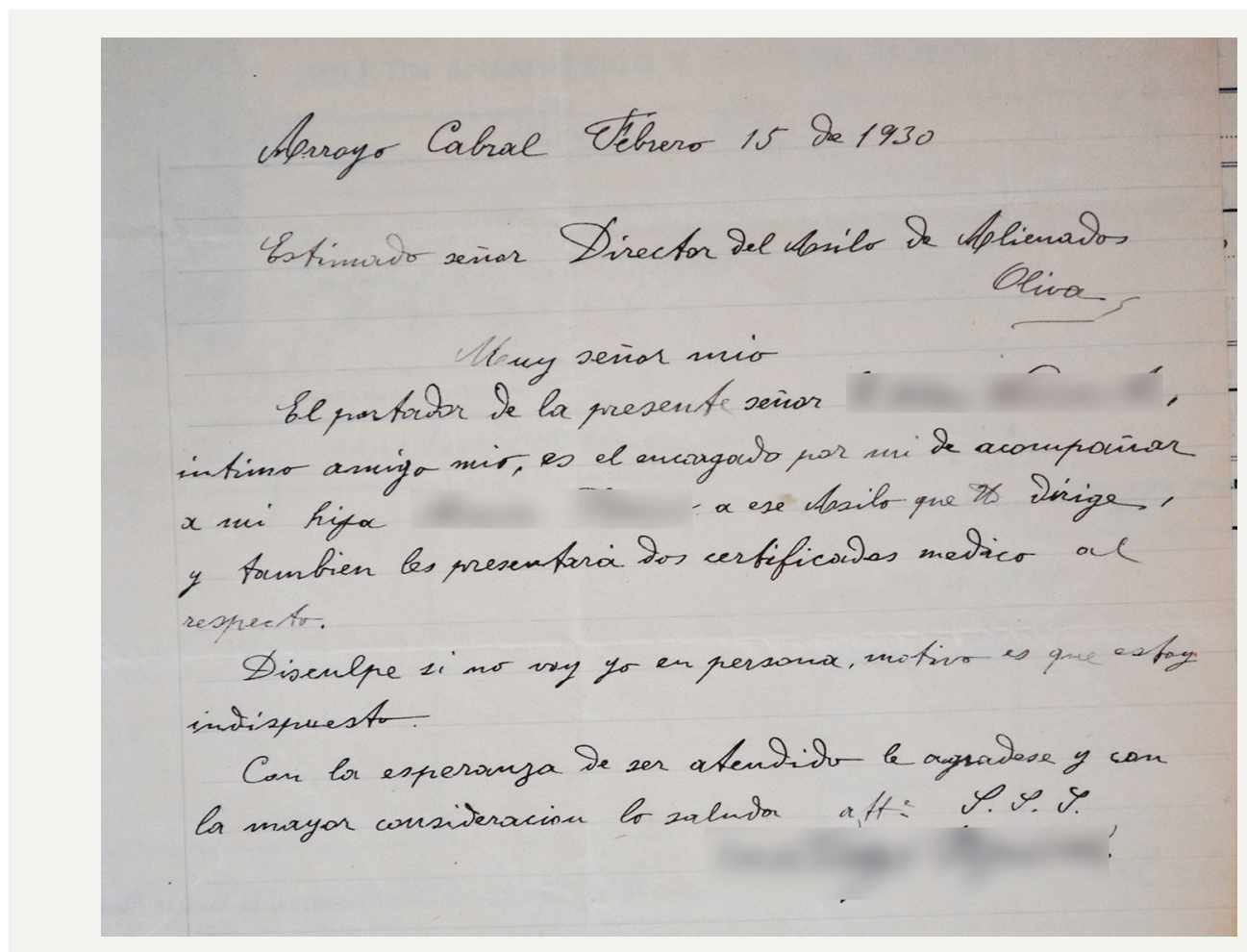

Figura 1. Fotografía de la carta de Antonio, presentada por su amigo Roque, solicitando la internación de Adela en el Asilo-Colonia Regional Mixto de Alienados de Oliva. 15 de Febrero de 1930. 
La experiencia analizada muestra hasta qué punto los ingresos se podían regir por usos y circunstancias de la vida social, las que constituirían una de tantas experiencias que usualmente debían resolver los médicos a cargo de las instituciones mentales. Asimismo, otros elementos evidenciaron que el mismo poder y legitimidad de los psiquiatras se hallaba lejos de constituir una autoridad consolidada. Hasta los renglones más formales del ingreso de Adela, asociados a la entrega que realizó Roque de dos certificados de alienación mental firmados por sendos médicos cirujanos, el Dr. Piñero y el Dr. Mendez, dan cuenta de esa inconsistencia. De acuerdo al Dr. Cubas, médico interno del Asilo de Oliva(18), la obligación de los psiquiatras de respetar esa "narración de síntomas" que, según él, cualquier médico estaba en condiciones de realizar, escondía una dramática situación asociada a que:

\footnotetext{
...en la mayoría de los casos [...] los certificados de alienación se extienden sin ver al enfermo. [...] [incluso] este cargo no es solo imputable a médicos particulares, sino que en muchos casos profesionales que desempeñan tareas oficiales, tales como médicos de policía, tribunales, etc., no tienen inconveniente en certificar un estado que no han comprobado, confiados solamente en la buena fe del acompañante, que si a veces es un familiar muchas veces es un extraño. (HC-AHEVA, 1929)
}

Aunque buscamos evitar adelantarnos en el análisis que pueden ofrecer los renglones dedicados al diagnóstico, la nosografía y los tratamientos, algunos puntos que se derivaron de la entrevista médica que se le realizó a Adela en 1930 son altamente reveladores. El sentimiento de tristeza y su idea de estar "nuevamente en Oliva" por no querer trabajar en su casa (HC-AHEVA, 1929) ponen en el centro de la situación los conflictos intrafamiliares de esta joven que, como mujer, se hallaría profundamente condicionada por las desigualdades de género imperantes. Ciertos puntos de esta historia solo pueden argumentarse a través de conjeturas, que remiten a preguntarse por la escasa libertad de elección de Adela para no desplegar las funciones primordiales que se esperaba de ella en el seno del hogar así como por el alcance del control patriarcal sobre el destino de la joven, en una época en que las mujeres aun eran consideradas por el Código Civil sujetos de incapacidad relativa, que dependían jurídicamente de los varones ${ }^{(19)}$. Asimismo, la insolvencia material de la joven fue manifiesta, evidentemente vinculada a la situación socioeconómica de su padre, tutor legal y proveedor de la vida material de su hija. Seguramente, el destino de aquel hombre fue muy distinto a su expectativa, al emigrar de su Italia natal en busca de mejores oportunidades promocionadas por los gobiernos de la época ${ }^{(3)}$. La vida de Antonio fue extraordinariamente complicada a causa de su alcoholismo, pero también por la precariedad laboral que implicaba trabajar de jornalero rural, seguramente de alguna empresa agrícola o chacra de Arroyo Cabral.

A pesar de que nuestros psiquiatras no integraron las relaciones interpersonales en sus nosografías y semiologías, algunas breves anotaciones consignadas en la historia clínica de la paciente, conceptuadas como "antecedentes hereditarios" (HC-AHEVA, 1929), revelaron que su madre y luego su padre también fallecieron internados en Oliva. Rosa murió en algún momento sin especificar entre en 1915 y 1930 -cuando ingresó Adela- complicada por las consecuencias de un alcoholismo avanzado, acompañado por un cuadro de "debilidad mental" (HCAHEVA, 1915). Antonio murió rápidamente también a causa de un alcoholismo avanzado apenas trascurridos 20 días desde su ingreso el 9 de noviembre de 1931 (HC-AHEVA, 1931). Destacamos que el primer ingreso de Rosa a Oliva sucedió en noviembre de 1914, fecha en que fue trasladada al recién inaugurado Asilo Colonia Regional Mixto de Alineados de Oliva desde el Hospital Nacional de Alienadas (Buenos Aires); probablemente esta mujer haya sido parte de aquel primer contingente de personas que se derivaron a Oliva ${ }^{(10)}$. Completando la estructura familiar, 
Adela tenía al menos cuatro hermanos que "vivían sanos" (HC-AHEVA, 1929); no obstante, la ausencia de ellos fue una constante, salvo en 1933, cuando a pedido de las autoridades de Oliva "los miembros de su familia" gestionaron ante las autoridades de Arroyo Cabral un "certificado de pobreza de solemnidad" para la interna (HC-AHEVA, 1929). Después de todo, Oliva estaba destinado, desde su creación, a la asistencia de indigentes ${ }^{(9)}$.

La localización de estas dos historias clínicas permitió proponer un análisis mucho más completo de las circunstancias de ingreso de la paciente, arrojando datos que colaboran en matizar aún más la idea de que la psiquiatría y sus instituciones, en este caso Oliva, sostuvieron de manera predominante una función de control social y disciplinamiento de los sectores pobres ${ }^{(9,20)}$. El hecho de que ambos padres -Rosa y Antonio- hubieran presentado síntomas orgánicos, definió que se los considerara enfermos agudos que requerían asistencia en cama, tratamientos ligados sobre todo a lo farmacológico y una vigilancia permanente. Consecuentemente, fueron destinados al área del hospital de Oliva, separada del área colonia-asilo, donde Adela pasó la mayoría de su vida. Este otro sector de la institución, que era el más extenso, estaba formado por grandes pabellones o villas -islas-, y era el espacio en el que residía la población de pacientes considerados crónicos pero inofensivos, tranquilos, en donde la terapéutica predominante y el eje de sostenimiento económico fue la laborterapia ${ }^{(9)}$.

Desconocemos detalles cruciales de la relación de este matrimonio pero, aun así, el deterioro fue evidente. Rosa había ingresado a Oliva llevada por la policía de Río Cuarto envuelta en un ataque contra su marido a quien acusaba de propinarle constantes maltratos (HC-AHEVA, 1915). Difícilmente se pueda entender que la mayoría de personas hospitalizadas en las instituciones psiquiátricas lo hacían engañadas o "secuestradas", pero esta realidad fue una preocupación de los psiquiatras argentinos. En sus términos $^{(18)}$, estas situaciones eran asociadas a intereses económicos y desidia de las familias, observándose dos prácticas bastante extendidas: que algunos familiares "hicieran pasar por locos" a sus parientes para encerrarlos y así quedarse con sus bienes, y que los internaran para no enfrentar el costo que traía consigo solventar la vida y convivencia "con un senil o un[a] idiota"(18).

Efectivamente, Adela y cada uno de sus padres representaron casos heterogéneos, tanto por las enfermedades diagnosticadas como por sus lógicas de ingreso. El ingreso voluntario de Antonio al hospital de Oliva completa el cuadro repasado. Tan grave y deteriorada habría sido su situación que, según manifestó quien lo acompañó desde su casa hasta Oliva, solo quería "...que le den algo para morir" (HC-AHEVA, 1931). Sin duda, la internación de Rosa en 1915, cuando nuestra joven apenas tenía cuatro años, y la convivencia con aquel padre alcohólico, explicarían muchos de sus comportamientos y padecimientos. Huelga decir que el cuadro con el que ingresó aquel italiano de 54 años, diagnosticado de alcoholismo orgánico con demencia (HC-AHEVA, 1931), no se había desarrollado en pocos meses, más bien habría acarreado años de tensiones y sufrimientos. La mirada del Dr. Fazio, interno de guardia que atendió en un primer momento a Antonio, refirió a su estado "psíquico", caracterizándolo por la incoordinación, los juicios absurdos y la desorientación completa con "...depresión intensa y desafectivisación familiar" (HC-AHEVA, 1931). En este marco, difícilmente podemos leer su "indisposición" aquel 15 de febrero -cuando mandó a Roque a internar a Adela- sin considerar la evolución previa de su patología.

La historia que sigue involucra más actores y procesos leídos desde un contexto más amplio, pero no por ello menos crucial para la experiencia de la paciente internada cuando, al aumento progresivo del grupo de alienados crónicos, se le sumó un panorama de recursos insuficientes que ocasionaría fisuras en el modo de funcionamiento del hospital-asilo de Oliva ${ }^{(9)}$. 


\section{SABER VER: SÍNTOMAS Y ETIOLOGÍAS}

Ese mismo 15 de febrero de 1930, el Dr. Barrancos dio a conocer el diagnóstico psiquiátrico de Adela, "depresión melancólica simple" (HC-AHEVA, 1929), una decisión que, aún con visos científicos, representaba ser una suerte de sentencia, si consideramos que la internación duró 38 años. Ahora, si avanzamos más cuidadosamente, notamos que esta perspectiva que asimila el diagnóstico a un poder discrecional está amparada en una ventaja del presente que descuida la configuración de los procesos cognitivos y los saberes que estuvieron en juego en esa época. El buen estado físico de Adela, es decir la función normal de su cuerpo, definió que su lugar dentro del espacio institucional fuera distinto que el de sus padres, comprometidos orgánicamente. Fue destinada al aislamiento dentro del área asilo, espacio que al menos en principio habría estado dedicado a los crónicos incurables.

Las fojas de la historia clínica de 1929 y de 1930 reiteran la etiqueta "depresión melancólica simple" (HC-AHEVA, 1929), diagnóstico usual en el universo de clasificaciones nosográficas de la época, aunque también bastante discutido en la psiquiatría europea ${ }^{(21)}$. Además de otras cuestiones que se repiten invariablemente en las historias clínicas de mujeres, como las relativas a las irregularidades/regularidades menstruales, en ambas fechas, podemos trazar similitudes y diferencias destacables en el estado observado en la paciente y en los criterios médicos articulados por Fazio y Barrancos. Aunque no interesa cuestionar la adecuación clínica del diagnóstico de estos médicos, sus registros habilitan discusiones sobre cómo se produjeron sendas interpretaciones, las que a fin de cuentas habilitaron el diagnóstico de Adela, hito inicial de sus posteriores itinerarios de internación en la institución. Desde esta perspectiva, conjeturamos algunas tensiones y trasformaciones que surcaron el saber/poder de estos psiquiatras durante el examen de los síntomas de la paciente, así como cuando procuraron explica- ciones etiológicas de la enfermedad mental que entendían que padecía la joven.

Un primer mojón lo constituyen las marcas presentes en la semiología construida durante la primera internación de Adela en Oliva, rubricadas por el Dr. Fazio. En aquella fecha, sus expresiones se asociaron a términos relativamente especializados tales como "abulia", "depresión" y "actitudes hipomaniacas", fundados en una lectura de síntomas que, en la observación del psiquiatra, se resumieron a que al ingreso, la paciente se encontraba "...tranquila, orientada, deprimida, cuesta mucho arrancarle una contestación, parece temerosa, cohibida, regularmente instruida" (HC-AHEVA, 1929). La renuencia o incapacidad de Adela para responder al interrogatorio de ingreso realizado por Dr. Fazio creó las condiciones para que las versiones de su padre fueran una pieza indispensable de la valoración psiquiátrica. Posiblemente por ello, la anamnesis en cuestión fue muy escueta, registrando, por un lado, las impresiones de Fazio y, por otro, las declaraciones de Antonio, quien manifestaba que "Hace aproximadamente un año comenzó a notarla [a su hija] cambiada en sus modalidades, deprimida, Ilorosa" (HC-AHEVA, 1929).

La insistencia en el poder estratégico de la palabra de Antonio hace que destaquemos la utilización de la expresión "depresión", cruzando los lindes académicos, término que Antonio posiblemente había incorporado por la larga trayectoria de su familia en instituciones psiquiátricas. De todas formas, no debemos confundir la importancia dada a la visión del padre con una significatividad otorgada a la palabra en sí misma. Este ingreso de Adela dio cuenta de la perduración en Oliva de estructuras y concepciones eminentemente tradicionales de corte anatómico-patológico, lo que se tradujo en la elaboración de una ficha craneométrica que detallaba la "morfología del cerebro", como podemos observar en la Figura 2, en línea con un "modelo somático" propio de la psiquiatría positivista de comienzos del siglo XX ${ }^{(22)}$. Desde esta dimensión fisiológica, el cuerpo aún vivo de Adela buscaba ser interrogado por Fazio como objeto de análisis de su alineación. 


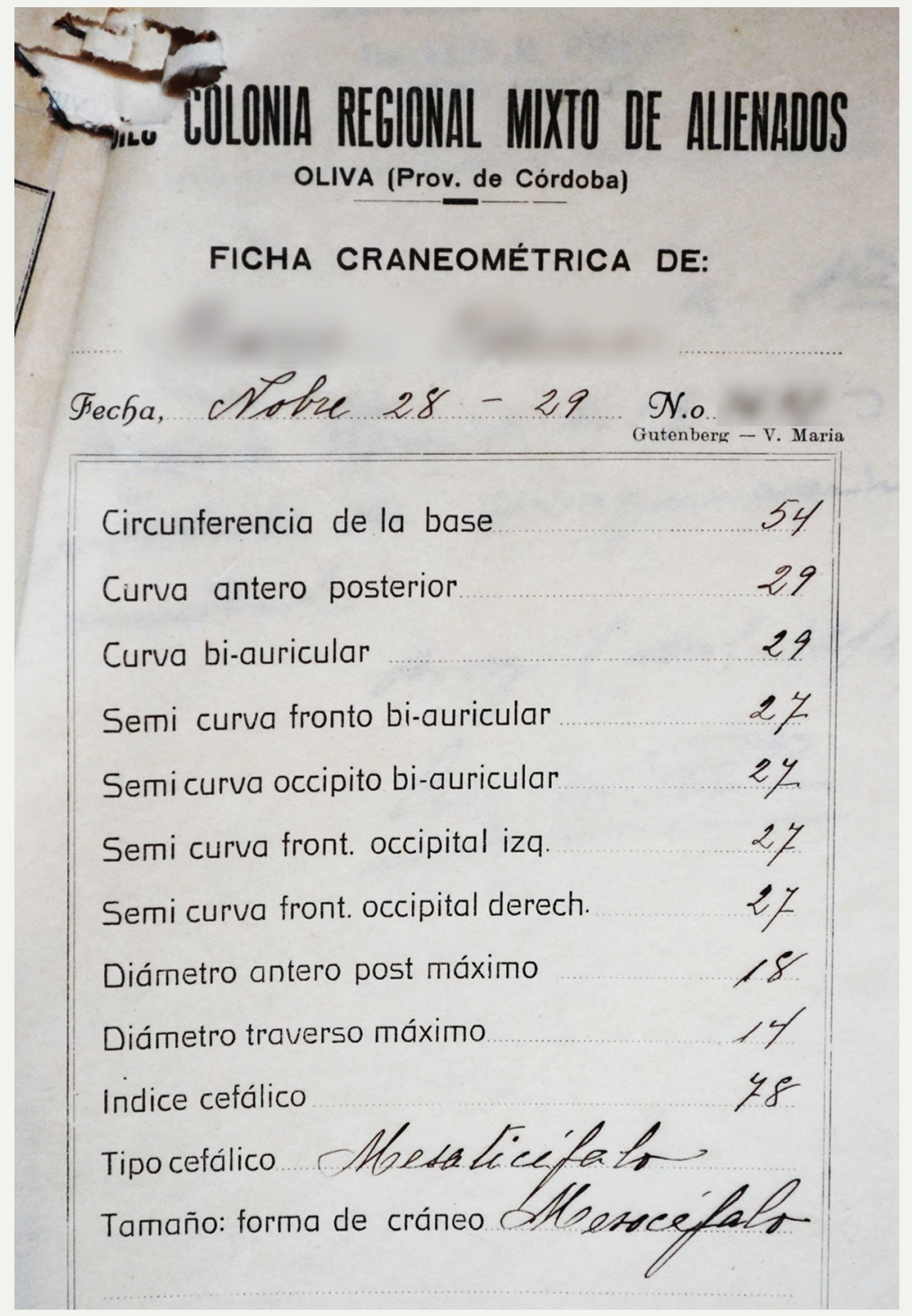

Figura 2. Fotografía de ficha craneométrica de Adela.

Fuente: Historia clínica, Archivo Hospital Emilio Vidal Abal.

Lo expresado en el párrafo anterior, si bien parece verificar la idea de Navarlaz ${ }^{(23)}$ sobre unas nosografías argentinas que continúan avalando hipótesis y utilizando herramientas centradas en causalidades orgánicas, este tipo de abordaje no impidió que unos días más tarde, fuera "...propuesta su alta", porque su "estado de depresión ha mejorado notablemente" (HC-AHEVA, 1929), lectura que implicaba la incorporación tácita de factores anímicos y volitivos.
En 1930, la recurrencia de síntomas se evidenciaba parcialmente. El registro efectuado en esa primera entrevista realizada a Adela, a cargo del Dr. Barrancos, se movió a partir de una narrativa mucho más "fenomenológica" de la conducta de la paciente, indicando que no mostraba señales visibles de desorientación o exaltación, se presentaba tranquila, temerosa, callada, bien orientada en espacio y tiempo, manifestando "...estar triste porque tiene muchas penas [...] contestando 
a las preguntas sin retardo, con coherencia" (HC-AHEVA, 1929), tendencia que no se había observado el 20 de noviembre de 1929, cuando a Fazio le costaba "...mucho arrancarle una contestación" (HC-AHEVA, 1929).

Barrancos abrió la posibilidad de acercarse a Adela de una manera distinta a lo que había practicado su colega. Ciertamente, aunque no podemos concluir que la palabra de Adela tuviera un efecto sobre la interpretación médica, fue novedoso en este caso que la entrevista a la paciente se orientara a cuestiones tan sensibles como las referidas a "la necesidad de alejar al paciente de la familia". Si bien los vacíos y silencios en la historia clínica dificultan enormemente la posibilidad de comprender renglones de por sí escurridizos de la clínica psiquiátrica, no fue menor que Barrancos registrara la sospecha sobre la causa de internación de Adela: "no querer trabajar en casa" (HC-AHEVA, 1929). A pesar de que la experiencia de la joven no escapaba a los estereotipos de género, esto no induce a pensar que su condición de mujer habría representado la clave que explicaría su condición de enferma mental y su larga internación y muerte dentro de Oliva, tal como ha sugerido $S y^{(24)}$ al estudiar las historias clínicas de mujeres migrantes alojadas en el hospital psiquiátrico de Lomas de Zamora para el mismo periodo que venimos abordando.

En cambio, fue patente el efecto que tuvo la porosidad del "saber ver" de Barrancos, permeable a las visiones y dichos de Roque, en detrimento de la palabra y subjetividad de Adela. Como había sucedido en noviembre del 1929, aunque por otras razones, las versiones del informante fueron decisivas al decir que Adela habría manifestado “...que desearía suicidarse (se envenenaría) pero nunca realizó tentativas" (HC-AHEVA, 1929). Desconocemos si existieron otros diálogos que no se plasmaron en los documentos correspondientes, pero nada indica que la ideación suicida fuera un tópico de conversación entre el médico y su paciente, a pesar de que tal asomo fuera crucial. Efectivamente, la gravedad de esta información fue perentoria, dado que las personas con intensiones suicidas eran especialmente conceptuadas como un peligro para sí mismas, problemática que los psiquiatras de Oliva asociaban al imperio del Código Civil que, en su artículo 156, marcaba que el demente no sería privado de su libertad personal, sino en los casos en que sea de temer que, usando de ella, se dañe a sí mismo o a otros ${ }^{(18)}$. Adela, para ese entonces ya había sido internada dos veces y nuevamente volvía a presentar certificados de alienación los que, si bien eran conceptuados como poco fiables, marcaban los límites legales entre los que se podían mover estos psiquiatras.

Las constricciones que venimos marcando representan una de las tantas facetas del alienismo y sus instituciones. El caso en análisis ofrece una mirada del manicomio y sus profesionales como un espacio y unos actores más complejos, destacándose su rol en la producción del saber ${ }^{25)}$. Solo así es entendible que, durante la década de 1930, la formación y producción académica de los psiquiatras de Oliva, ampliamente difundida a través de la publicación de su revista Boletín del Asilo de Alienados en Oliva durante los años 1933-1942, sobreviniera en un foco de atención para los psiquiatras nacionales y extranjeros ${ }^{(9)}$, proceso que convivió inestablemente con aquel crítico panorama de recursos humanos y financieros que hemos apuntado. Estos asuntos también se vinculaban a búsquedas de construcción de legitimidad para la especialidad y su entorno de trabajo.

Las prácticas científicas del Dr. Barrancos convirtieron la enfermedad de Adela en un objeto de estudio privilegiado, integrándola como parte de una investigación, compuesta por una muestra de 82 casos de "padres e hijos" que estaban o habían estado internados en Oliva, a partir de los cuales se proponía sacar "conclusiones" sobre "el asunto de la herencia de las enfermedades mentales"(26) (Figura 3). Como hemos adelantando, la cuestión del alcoholismo y los "antecedentes hereditarios" de Adela fueron piezas destacadas en la estructura dedicada al ingreso de la paciente.

Tomando como referencia los modelos somáticos que imperaron en la psiquiatría 


\section{XXVII}

58. - ( $)-1$, itallano, casado, jornalero, 5 f años alcoholista, ideas melancolicas, pide algo para morirse tranquilo. desorientado, incoherente, gatismo, ictus hemorrágico. Demencia orgánica.

59. - ( ) - $=0$, !ija del anterior: argentina, soltera. 19 años; ingresa en 1929. Deprínida, temerosa. Jlorosa. callada bien orientada, desearía suicidarse pero nunca lo ha inten. tado. Dice tener "mucha pena". laeas hipocondriacas. Depresión melancólica simple.

Figura 3. Fotografía de la cita del caso de Adela y su padre en publicación científica del boletín institucional.

Fuente: Barrancos ${ }^{(26)}$.

argentina hasta la década de $1920^{(22)}$, de la cual Fazio fue un representante de caso, distintos renglones de la investigación de Barrancos sugieren la incorporación de algunas transformaciones en los modelos explicativos de las enfermedades mentales producidas en y para los alienados del asilo de Oliva. En términos generales, su aporte puede asociarse al tránsito hacia una concepción de enfermedad mental permeable a la injerencia de factores explicativos de tinte social, abandonando el determinismo positivista que aún se imponía en muchos psiquiátricos de la época ${ }^{(22)}$.

Como el mismo médico hizo notar, en la clínica psiquiátrica lo que se “...hereda no es verdaderamente la enfermedad sino una predisposición" [y] "la predisposición, por sí sola no basta. Forzosamente, habrá que agregar la intervención de otros agentes, tóxicos, ambientales, infecciosos, etc."(26). Ahora bien, a pesar que para este psiquiatra "El enfermo es, en cierto modo, un reflejo del ambiente en que vive o ha vivido"(26), sus planteos no están abiertos a la incorporación de las corrientes psicodinámicas para las cuales los conflictos intrapsíquicos devenían en elementos explicativos fundamentales. En ese sentido, su referencia a Freud en alusión a las influencias de las condiciones externas que el alemán "...hace remontar a la edad infantil" (26), estaría abonando la idea de una imprecisa modernización de la psiquiatría argentina en transformación ${ }^{(22)}$, permeable a incorporar "nuevos" modelos e hipótesis para entender el origen de ciertas enfermedades.
Asimismo, y a pesar de que su preocupación no estaba focalizada en Adela y en los otros enfermos de la muestra como sujetos de padecimientos, sino más bien en la enfermedad como entidad nosográfica, son de primer orden sus contribuciones en cuanto a reposicionar el rol del espacio social entre las causas de la enfermedad mental, junto a su insistencia en la necesidad y posibilidad de articular una profilaxis que, en sus términos, apareció vinculada a la internación temprana. En efecto, el peso de su trama argumentativa genera las condiciones para poner en cuestión la dominancia de un pesimismo terapéutico, que tanto ha avalado la historiografía sobre instituciones psiquiátricas. Aquella matriz que identificaba a los pacientes agudos con la posibilidad de cura y a los crónicos con la incurabilidad y su confinamiento al asilo y la asistencia social, estuvo sujeta a trasformaciones a través del tiempo y a las distintas lecturas según los psiquiatras y sus influencias. En ese sentido podemos leer la contribución del Dr. Bringas Núñez ${ }^{(27)}$, en 1941, sobre la relación entre ingresos y egresos en Oliva, en la que enfatizaba que las afecciones como las distímicas -que padecía Adela- no respondían necesariamente a “... procesos crónicos, aumentando las posibilidades de curación, en razón de que en tales condiciones la eficacia del tratamiento es mayor [y] en consecuencia los egresos crecen, disminuyendo entonces el pasivo"(27). 


\section{SABER HACER: LABORTERAPIA, CRONIFICACIÓN Y MUERTE}

Si las ideaciones suicidas eran relevantes en el caso de Adela y, frente a ellas, la institución se erigía en el espacio indicado para cuidar a la paciente de sí misma, las dificultades del asilo de Oliva para vigilar esta situación no eran poco importantes. Resulta muy consistente la imagen que se desprende como consecuencia de la reorganización emprendida en Oliva para 1926, cuando a la división existente entre las áreas de hospital y asilo, se le sumó una redistribución de los siete psiquiatras que trabajaban allí, destinando seis a la atención de los casos agudos (hospital) quedando tan solo uno, el director, a cargo del área del asilo-colonia que albergaba por entonces a 2.400 alienados $\operatorname{crónicos}^{(28)}$. Difícilmente esta situación nos devuelva una idea del poder de los alienistas sobre los cuerpos alojados en el asilo. Como apuntó Eraso, la proporción de médicos/alineados era "...en el año 1930, 1/452 $y$, en cuanto al personal de vigilancia diurno de 1/31alienados y en los serenos de 1/97 alienados $^{\prime \prime(9)}$.

Fue tan decisiva la importancia otorgada a aquella matriz organizacional hospital/asiloagudos/crónicos que, a partir de ella, se buscó leer todo el funcionamiento manicomial. De acuerdo a esto, el enfermo mental, especialmente "el crónico incurable", dirá Eraso ${ }^{(7)}$, se convirtió en el destinatario privilegiado del discurso psiquiátrico sobre el trabajo, y hacia la década de 1930, se concibe “...su posición no ya desde la concepción estrictamente psiquiátrica, sino desde la social-asistencial"(7). En efecto, los discursos médicos de estos años avalan constantemente la necesidad de expandir la laborterapia por motivos de rédito material en un marco institucional fustigado, preocupación que presentaban en sus publicaciones científicas, como una respuesta útil y necesaria para afrontar la situación: “...existe solo un método que llena todas las exigencias médicas, sociales, económicas y éticas: la laborterapia aplicada con el doble criterio científico y administrativo"(29).
Si bien es innegable que, como sucedía en otros psiquiátricos de la época, la aplicación de la laborterapia operó como una respuesta a los insuficientes recursos disponibles para sostener a estas instituciones públicas $^{(7,25)}$, ello no excluye que la laborterapia respondiera a una determinada concepción psiquiátrica. La historia clínica de Adela enseña el poliforme sentido que adquirió la internación y la terapia. No se podría poner en duda el valor económico del trabajo de Adela durante casi cuatro décadas, ni las constantes intervenciones sociales y asistenciales que fueron orientadas a sostener su estado de salud física general, consistentes básicamente en la administración de vacunación periódica, incluso de vitaminas, tratamientos dentales e internaciones varias y atención de coyunturas médicas por intoxicación con alimentos, picaduras de insectos, problemas digestivos, etc., (HC-AHEVA, 1929).

Sin embargo, dicha impronta no exceptuó cierto orden terapéutico en la asignación de tareas ocupacionales para Adela, que coincidían al menos en parte con debates disciplinarios que ocupaban la agenda de la psiquiatría en materia de tratamiento de enfermos mentales asilados ${ }^{(29,30,31)}$. En ese sentido, en la historia clínica observamos numerosos desplazamientos institucionales acompañados de cambios en las asignaciones ocupacionales, que respondieron a una idea compatible con el principio de complejización progresiva de las tareas, claro que de acuerdo al estado mental que fue mostrando la paciente.

Advertimos una secuencia tanto a nivel de sus traslados espaciales en las villas de la sección del asilo, como así también en cuanto a las tareas laborales que desarrolló. Tales series se inician con su desplazamiento a villas próximas al hospital -como Villa 13 (año 1930), Villa 16 (año 1946), Villa 14 (año 1961) en las que se desempeñara como mucama y como serena, seguido de desplazamientos a villas más lejanas -Villa 19 (año 1961), Villa 20 (año 1955), Villa 21 (años 1931, 1956, 1959, 1966)-, en las cuales las actividades labores exigían una mayor autonomía y estabilidad de Adela, y en las que se desempeñó en la verdulería, como mucama 
en el laboratorio de rayos $\mathrm{X}$ y otros talleres laborales específicos (HC-AHEVA, 1929).

También las publicaciones científicas sobre la laborterapia, difundidas desde el Boletín de Oliva avanzaron hacia un marco más científico y técnico que filantrópico. Sin desvincularse completamente de los fundamentos del tratamiento moral focalizado en el orden manicomial y la disciplina, los aportes locales discutieron e integraron postulados de nuevas orientaciones de profilaxis mental, como los anclados en el método que evitaba el derrumbe de la personalidad, aggiornándose con nuevas herramientas ${ }^{(29)}$. Una de ellas fue la terapia familiar, entendida como el trabajo del alienado en casa de familias de médicos de Oliva, de la que Adela también formó parte en repetidas oportunidades (años 1944, 1945 y 1959).

A mediados de la década de 1930, desde el Boletín institucional se fue prestando especial atención al afianzamiento de un discurso psiquiátrico centrado en la renovación de la terapéutica por el trabajo. Nos referimos a los desarrollos del Dr. Simon Hermann, psiquiatra somaticista alemán, que ensayó su método de praxiterapia con crónicos, volviendo a colocar el trabajo en el centro de la organización manicomial, a partir de insistir en la relevancia de la adaptación del paciente al medio institucional laboral y ofrecer una gran variedad de ocupaciones a asignar de manera progresiva, según nivel de complejidad creciente $^{(32)}$. Recordemos que el "tratamiento moral" ocupaba un lugar secundario tras su fracaso en Europa, cuando los propios creadores del alienismo tuvieron que enfrentar problemas para aplicarlos, como consecuencia de la realidad de los espacios de reclusión ${ }^{(33)}$.

De todas formas, los esfuerzos de teorización de los psiquiatras de Oliva no estuvieron exentos de críticas por parte de alienistas latinoamericanos, algunos de los cuales, como el Dr. Hernani Lopez (Brasil), ponían en duda que en Oliva se aplicara adecuadamente las concepciones terapéuticas en boga ${ }^{(34)}$. Tal como respondió el Dr. Ferrer, estas críticas no parecieron desmesuradas ya que, según se admitió, los límites vernáculos respondían a razones de distinto orden "...falta de personal suficiente, falta de recursos para la instalación de talleres de rendimiento exiguo, modalidades raciales, nivel cultural del tipo de población que ingresa, ausencia de gabinetes psicotécnicos, escaso números de médicos, etc., etc."(29).

De hecho, al contextualizar los ingentes resultados que acompañaron su tratamiento -si es que consideramos que el éxito se identifica con el alta médica que, como sabemos, nunca llegó- dos asuntos atravesaban la internación de Adela. Por una parte, los múltiples obstáculos que jalonaban las prácticas médicas, cuestión agravada a partir de los años en que la paciente ingresó, dado el engrosamiento progresivo de alienados crónicos provenientes de los hospicios de Buenos Aires ${ }^{(10)}$. Por otra, las particularidades del caso en análisis otorgaron un tono característico a la propia experiencia terapéutica: el registro de distintos comportamientos, leídos como síntomas -agitación, desorientación y delirio- fueron recortando las posibilidades de proponer el alta, habilitando otros tratamientos como la administración de bromuro e hidroterapia, terapias que si bien se aplicaron en este caso en la década de 1940, su uso en el entorno científico había sido desplazado tiempo antes como lo indican reconocidos análisis del alienismo argentino ${ }^{(10)}$.

Las marcas del deterioro físico y psicológico de Adela comenzaron a parecer irreversibles a partir de la década de 1950. Desde ese momento se registraban las huellas de su "desocialización" e institucionalización/ cronificación, en un proceso que fue desde "No desea volver a su hogar" (1951), "no desea irse de alta" (1965), "no recibe ninguna comunicación de la familia [y] se presume que ya no tiene a nadie que se interese por ella" (1965), adaptada al ambiente (1967), náuseas y vómitos (1969), y finalmente su muerte por un infarto dos meses más tarde (HC-AHEVA, 1929).

\section{CONSIDERACIONES FINALES}

El prolongado tiempo que duró el aislamiento de Adela en el asilo de Oliva da fuerza a 
aquellas lecturas que interpretaron el funcionamiento manicomial, enfatizando su carácter custodial, ligado a la preeminencia de la asistencia social del alienado crónico y la ineficacia terapéutica de la psiquiatría alienista. Ciertamente, Adela en sus más de tres décadas de internación fue objeto privilegiado de cuidados de tipo social, acompañados por breves intervenciones en el hospital. Claro que estas prácticas constituyeron solo una faceta de las múltiples experiencias que encerró Oliva, vistas desde el caso de Adela.

El análisis de las historias clínicas y de distintos artículos publicados en el Boletín institucional han descubierto cómo los psiquiatras fueron productores de saber, en parte envueltos en un compromiso científico y en parte movilizados por legitimar una profesión puesta en cuestión. Destacamos las posiciones e influencias teóricas del Dr. Barrancos en un contexto de trasformaciones en los modelos deterministas de explicación de las enfermedades mentales y su abordaje. Aunque estas investigaciones recrearon el tradicional interés alienista por los asuntos etiológicos sobre los semiológicos, sus aportes abrieron asuntos de primer orden, como los referidos al valor de la internación temprana y la entidad de los factores sociales como causa de los trastornos mentales.

Asimismo, la observación de una praxis clínica concreta evidenció la porosidad del conocimiento especializado, identificando cómo las miradas y versiones de la familia y los allegados permearon decisiones médicas cruciales para la trayectoria de nuestra paciente. En el caso de Adela, la influencia de los informantes externos no se redujo a una experiencia excepcional. Las situaciones y conflictos familiares que enmarcaron su ingreso e internamiento y su vulnerabilidad como joven mujer exhiben rasgos comunes a la sociedad de su época, asuntos que habrían estado exacerbados en la historia de una familia desintegrada en sus vínculos y afectada por el alcoholismo y la precariedad socioeconómica de su padre inmigrante. A esta altura no resulta excesivo pensar a Oliva como espacio de refugio y supervivencia. De todas maneras, sin restarle importancia al sufrimiento de Adela, los escasos recursos financieros y humanos con los que contaba la institución no habrían posibilitado que el ambiente de asilo se convirtiera en un lugar de vigilancia y disciplina contaste, como se prefiere ver desde los enfoques del control.

Lo anterior no nos impide suponer que la aplicación de la laborterapia fuera un engranaje de financiamiento institucional. Sin embargo, la historia clínica de Adela expone que la aplicación de esa terapia respondió a cierto orden con criterio científico. La población de alienados crónicos, y entre ellos nuestra paciente, no permanecieron en una zona de olvido e invisibilidad. La laborterapia habilitó un genuino espacio de reflexión y discusión académica, la cual visibilizó señeros cuestionamientos sobre el rol y las posibilidades del alienista, sino de "curar", al menos de "tratar", buscando recuperar la enfermedad mental. 


\section{REFERENCIAS BIBLIOGRÁFICAS}

1. Novick S. Población y Estado en Argentina de 1930 a 1943: Análisis de los discursos de algunos actores sociales: industriales, militares, obreros y profesionales de la salud. Estudios Demográficos y Urbanos. 2008;23(2):333-373. doi: 10.24201/ edu.v23i2.1314.

2. Rapoport M. Mitos, etapas y crisis en la economía argentina. Nación-Región-Provincia en Argentina. $2007 ;(1): 9-28$.

3. Rocchi F. El péndulo de la riqueza: la economía argentina en el periodo1880-1916. En: Mirra ZL, (dir.). Nueva Historia Argentina: El progreso, la modernización y sus límites, 1880-1916. Buenos Aires: Sudamericana; 2000. p. 15-69.

4. Ferrari F. De la locura a la enfermedad mental, Córdoba, 1758-1930: una historia cultural sobre los discursos y prácticas médicas sobre la locura. Córdoba: Alethéia Clío; 2016.

5. Falcone R. Genealogía de la locura: Discursos y prácticas de la alienación mental en el positivismo argentino (1880-1930). Buenos Aires: Letra Viva; 2012.

6. Vezzetti H. La locura en la Argentina. Buenos Aires: Folios Ediciones; 1983.

7. Eraso Y. El trabajo desde la perspectiva psiquiátrica: Entre el tratamiento moral y el problema de la cronicidad en el manicomio de Oliva de Córdoba en las primeras décadas del siglo XX. Cuadernos de Historia: Serie Economía y Sociedad. 2002;(5):33-63.

8. Iacoponi L. El Hospital Interzonal Colonia Dr. Domingo Cabred y el método Open Door. Alcmeon. 1999;7(4):5-14.

9. Eraso Y, Agüeros N. Trabajo alienado: Aportes para la comprensión del trabajo de los enfermos mentales en una institución psiquiátrica pública: Asilo Colonia Regional Mixto de Alienados en Oliva, 1914-1934. Córdoba: Escuela de Historia, Facultad de Filosofía y Humanidades, Universidad Nacional de Córdoba; 1999.

10. Ablard J. Madness in Buenos Aires: Patients, psychiatrists, and the Argentine State, 1880-1983. Calgary: University of Calgary Press; 2008.

11. Vanadia LN. Miradas del Hospital Asilo de Oliva, durante la primera mitad del siglo XX: algunas reflexiones teórico metodológicas para su análisis histórico. Revista de Salud Pública. 2017;21(3):82-87. doi: 10.31052/1853.1180.v21. n3.18894.
12. Sacristán C. Curar y custodiar: La cronicidad en el Manicomio La Castañeda Ciudad de México, 1910-1968. Asclepio. 2017;69(2):193-207. doi: 10.3989/asclepio.2017.16.

13. Venancio A, Saiol J. El Hospicio Nacional de Alienados en la prensa de Río de Janeiro (19031911). Asclepio. 2017;69(2):190-201. doi: 10.3989/ asclepio.2017.13

14. Gauchet M. De de Pinel a Freud. En: Swain G. Le sujet de la folie: Naissance de la psychiatríe. Paris: Calmann Lévy; 1997.

15. Stagnaro J. Evolución y situación actual de la historiografía de la psiquiatría en la Argentina. Frenia. 2006;6:7-37.

16. Sacristán C. La locura se topa con el manicomio: una historia por contar. Cuicuilco. 2009;16(45):163-189.

17. Huertas R. Las historias clínicas como fuente para la historia de la psiquiatría: posibles acercamientos metodológicos. Frenia, Revista de Historia de la Psiquiatría. 2001;1(2):7-37.

18. Cubas M. Los certificados médicos de alienación mental. Boletín del Asilo de Alienados de Oliva. 1935;3(7):35-45.

19. Giordano V. (Doble) moral sexual y derechos civiles de las mujeres, 1888-2010. En: Barrancos D, Guy D, Valobra A. Moralidades y comportamientos sexuales: Argentina, 1880-2011. Buenos Aires: Editorial Biblos; 2014.

20. Dovio M. La "mala vida" y el Servicio de Observación de Alienados (SOA) en la revista Archivos de PCMLyCA (1902-1913). Sociológica. 2011;26(74):79-108.

21. Berrios G. Historia de los síntomas de los trastornos mentales: la psicopatología descriptiva desde el siglo XIX. México: Fondo de Cultura Económica; 2013.

22. Falcone R. Historia de las prácticas de la psicología, la psiquiatría y el psicoanálisis: Resultados de la investigación a partir de casos clínicos (19001960). Anuario de Investigaciones. 2013;20.

23. Navarlaz VE. La hipótesis de la degeneración en las historias clínicas del Hospicio de las Mercedes y la Colonia Dr. Cabred entre los años 1900 y 1930. Anuario de Investigaciones. 2009;16:183-191.

24. Sy A. Mujeres, migrantes y "locas": Trayectorias de internación psiquiátrica entre 1895 y 1940 en Argentina. TraHs, Trayectorias Humanas Trascontinentales. 2018;(3):5-19. doi: 10.25965/trahs.754. 
25. Campos Marín RA, Huertas R. Los lugares de la locura: reflexiones historiográficas en torno a los manicomios y su papel en la génesis y el desarrollo de la psiquiatría. Arbor. 2008;184(731):471480. doi: 10.3989/arbor.2008.i731.197

26. Barrancos A. Padres e hijos alienados. Boletín del Asilo de Alienados de Oliva. 1935;3(10):288.

27. Bringas Núñez E. Bases para la creación de un establecimiento provincial para alienados en Córdoba. Boletín del Asilo de Alienados de Oliva. 1941;9(39):271-290.

28. Vidal Abal E. El Asilo de Oliva. Boletín del Asilo de Alienados de Oliva. 1933;(1):5-12.

29. Ferrer C. Nuevas consideraciones sobre el trabajo en los alienados. Boletín del Asilo de Alienados de Oliva. 1934;(4):77-91.
30. Vidal Abal E. Sobre la asistencia al alienado. Boletín del Asilo de Alienados de Oliva. 1935;3(8):95-98.

31. Hernández Ramirez R. El trabajo como terapéutica de la locura. Boletín del Asilo de Alienados de Oliva. 1934;(5):194-200.

32. Simon H. El tratamiento ocupacional en los enfermos mentales. Barcelona: Salvat; 1937.

33. Huertas R. Del manicomio a la salud mental: Para una historia de la psiquiatría pública. Madrid: FISS; 1992

34. Lopez H. Algunos comentarios sobre la laborterapia del Hospital de Oliva. Archivos Brasileiros de Hygiene Mental. 1933;6(4):316-324.

FORMA DE CITAR

Vanadia LN, Rodríguez ML. El saber ver y el saber hacer en el hospital-asilo de Oliva (1914-1950): el caso Adela. Salud Colectiva. 2019;15:e2211. doi: 10.18294/sc.2019.2211.

Recibido: 1 de marzo de 2019 | Versión final: 12 de julio de 2019 | Aprobado: 16 de julio de 2019

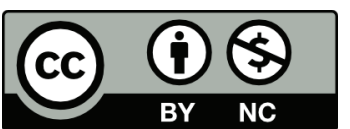

Esta obra está bajo una licencia de Creative Commons Reconocimiento-NoComercial 4.0 Internacional. Reconocimiento - Permite copiar, distribuir y comunicar públicamente la obra.

A cambio, se debe reconocer y citar al autor original. No Comercial - Esta obra no puede ser utilizada con finalidades comerciales, a menos que se obtenga el permiso. 